Radial and Nonradial Pulsations as Probes of Stellar Physics

ASP Conference Series, Vol. 259, 2002

C. Aerts, T.R. Bedding, \& J. Christensen-Dalsgaard, eds.

\title{
Aperiodic Ordered Sequences and Multiperiodicity in Semiregular Variable Stars
}

\author{
Juan García Escudero \\ Departamento de Física, Universidad de Oviedo, 33007 Oviedo, Spain
}

\begin{abstract}
The use of aperiodic ordered sequences described by Lindenmayer systems in the study of the strings of periods for multiperiodic semiregular variables is proposed. Fibonacci sequences play a central role in the light curve analysis of UW Herculis.
\end{abstract}

\section{Introduction}

Aperiodic ordered structures have been considered during the last two decades for the study of a new type of material known as quasicrystals. Deterministic and stochastic models have been investigated (Escudero, 2000). Scale invariance is one of their properties. It is a more general concept than fractality and has been used for the study of distribution of galaxies (Balian, 2000). Selfsimilar aperiodic sequences in 1D, which lie somewhere between periodicity and randomness and their Fourier analysis, are the main tools of our study. The scaling orinflation factors of the sequences are algebraic integers. The rate of periods of multiperiodic semiregular variables like ST Cam, V Boo or UW Her (Kiss et al., 1999) are close to algebraic integers. The Fourier analysis of a Fibonacci quasiperiodic temporal sequence of sinusoidal fragments can be characterized by a module consisting of integer linear combinations of two fundamental frequencies in a golden ratio. The module is a dense set in the Fourier space, but above a certain threshold the number of peaks below a given frequency is finite. The frequencies with higher amplitudes in the light curve analysis of UW Her can be considered as part of the Fourier module of Fibonacci sequences. The analysed data belong to the BAA Variable Star Section archive.

\section{Fibonacci sequences.}

Lindenmayer systems can be used in order to describe the Fibonacci sequences. A0L-system (Prusinkiewicz \& Lindenmayer, 1990) is a triple $G=\{\Sigma, r, \omega\}$ where $\Sigma$ is an alphabet, $r$ is a finite substitution on $\Sigma$ into the set of subsets of $\Sigma^{*}$, and $\omega$ is the axiom. G is called a D0L-system if $\#(r(x))=1$, for every $x \in \Sigma$. For the Fibonacci sequence the alphabet is $\{L, S\}, r:\{L \longmapsto L S, S \longmapsto L\}$ and the axiom $L$. The sequence consists in the words $L, L S, L S L, L S L L S, \ldots$ A $1 \mathrm{D}$ quasiperiodic geometric structure can be obtained if $L$ and $S$ represent two segments with a ratio equalling the golden number $\tau$.

Consider artificial data generated by concatenation of two sinusoidalfragments with lengths $L=89$ and $S=55$. The Fourier analysis of the se- 
quence $r^{9}[L]$ shows that the peaks are situated on the Fourier module $f_{m, n}=$ $\Sigma\left(m f_{1}+n f_{2}\right)$ where $m, n \in Z$ and $f_{1} / f_{2}=\tau$. The frequencies with highest intensities $f_{1}$ and $f_{2}$ correspond to the periods 123 and 76 . Observe that there is a factor of 1.38 in relation to the two periods forming the data. The existence of sharp peaks, or discrete component, in the Fourier spectrum is related to the fact that $\tau$ is a Pisot number.

Astochastic 0L-system is a 4-tuple $G=\{\Sigma, P, \omega, \pi\}$ where $P$ is a set of productions $r_{i}$ and $\pi: P \longmapsto(0,1]$ is a probability distribution. We define a Fibonacci stochastic L-system with $P=\left\{r_{1}, r_{2}\right\}$ and $r_{1}:\{L \longmapsto L S, S \longmapsto$ $L\}, r_{2}:\{L \longmapsto S L, S \longmapsto L\}$. The Fourier spectrum of artificial data generated by following the word sequences of this system contains peaks with bandwidths due to the presence of a continuous component.

\section{The multiperiodic semiregular UW Her.}

The Fourier analysis of the light curve of UW Her between JD 2442152 and 2451661 shows that the peaks with highest intensities belong to the Fourier module of a Fibonacci sequence. The three most significant periods of the analysis are $p_{1}=107, p_{2}=173$ days and a double peak in 1057 and 1119 days. Although the time span of the data set is different, the results are in good agreement with that of Kiss et al. (1999) except for the longest period, which could be explained by its instability. For the period between MJD 6154 and 9814 (MJD=JD-2441799) the Fourier spectrum of the visual data shows that the three most intense peaks correspond to $p_{1}=107, p_{2}=174$ and $p_{3}=1220$ days. A model can be obtained with the help of a Fibonacci stochastic L-system with $L=126$ and $S=78$ days. The following derivation corresponds to the period between MJD 7552 and 9814: $L \longmapsto S L \longmapsto L L S \longmapsto S L S L L \longmapsto L L S L L S L S \longmapsto S L L S L S L L S L L S L \longmapsto$ LLSLSLLSLLSLSLSLLSLSL . For the model corresponding to MJD 61549814 the highest intensities in the Fourier analysis correspond to $p_{1}=107$ and $p_{2}=174$ days, if all the sinusoidal fragments have the same amplitude. If the amplitudes of the sinusoidal fragments with maxima situated on MJD 7081, 8479 and 9649 are increased, then the peak corresponding to $p_{3}=1220$ appears in the spectrum.

Acknowledgments. This work has been done with the assistance of the BAA Ridley Grant. I thank variable-star observers of BAA whose magnitude estimates over the years made this study possible.

\section{References}

Balian, R. 2000 in From Quasicrystals to more complex systems, (EDP-Springer), 13,329

Escudero, J.G. 2000, Mat. Sci. Eng. A, 294, 388

Kiss, L.L, Szatmáry, K., Cadmus, R.R., \& Mattei, J.A. 1999, A\&AS, 346, 542

Prusinkiewicz, P. \& Lindenmayer, A. 1990, The Algorithmic Beauty of Plants (Springer) 